Annales Geophysicae (2001) 19: 589-592 (c) European Geophysical Society 2001

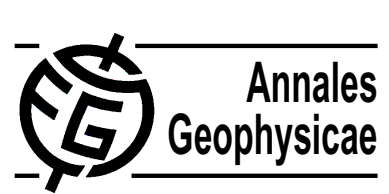

\title{
Astrid-2, an advanced microsatellite for auroral research
}

\author{
G. T. Marklund ${ }^{1}$, L. G. Blomberg ${ }^{1}$, and S. Persson ${ }^{2}$ \\ ${ }^{1}$ Alfvén Laboratory, Royal Institute of Technology, SE 10044 Stockholm, Sweden \\ ${ }^{2}$ Swedish Space Corporation, Solna, Sweden
}

Received: 3 July 2000 - Revised: 9 October 2000 - Accepted: 16 October 2000

\begin{abstract}
The successful launch of the Swedish microsatellite Astrid-2 in December 1998 began a new era of auroral research, with advanced microprobes of $30 \mathrm{~kg}$ or less used as research tools. Innovative technologies and low-mass solutions were used for the sensors and deployment systems to allow a fairly complete set of scientific instruments within the $10 \mathrm{~kg}$ allocated for the scientific payload. A newly developed wire boom deployment system proved to function excellently. During its seven month lifetime Astrid-2 collected more than 26 Gbytes of high-quality data of auroral electric and magnetic fields, and auroral particle and plasma characteristics from approximately 3000 orbits at an inclination of $83^{\circ}$ and an altitude of about $1000 \mathrm{~km}$. Scientific results cover a broad range of topics, from the physics of energization of auroral particles to how the magnetosphere responds to the energy input from the solar wind and global magnetic field modelling. The fulfilment of both the technological and the scientific mission objectives has opened entirely new possibilities to carry out low-budget multipoint measurements in near-Earth space.
\end{abstract}

Key words. Ionosphere (auroral ionosphere; instruments and techniques) - Magnetospheric physics (auroral phenomena)

\section{Introduction}

As a continuation of the successful Swedish small-satellite programme, which produced Viking, 1986 (Hultqvist, 1990) and Freja, 1992 (Lundin et al., 1994a, b), a microsatellite programme was embarked upon in 1993. One of the ultimate goals of the programme was to facilitate inexpensive missions for auroral research, and as a further step, to enable multipoint auroral missions. The first microsatellite in the Astrid series, Astrid-1, was launched in January 1995, carrying an energetic neutral particle imager as its main payload. The flight was successful, both in demonstrating that neutral

Correspondence to: G. T. Marklund

(marklund@plasma.kth.se) particle imaging is a powerful research tool and in serving as a proof of concept for a scientific microsatellite (Barabash et al., 1997; 1998; C:son Brandt et al., 1999; Norberg et al., 1995)

Inspired by the success of Astrid-1, the second spacecraft in the series, Astrid-2 (Marklund et al., 1997; Blomberg et al., 1999), was given a project go-ahead in the spring of 1995. Astrid-2 carried a much more comprehensive payload than Astrid-1, requiring a longer development time but still within a very low budget ( 2.5 MUSD including all costs for the satellite platform, launch, and mission operations but excluding costs for instrument development and data analysis). Astrid-2 was focused on diagnosing auroral processes in the Earth's ionosphere and upper atmosphere. In addition to its scientific objective, Astrid-2 also had a technological objective, namely to demonstrate the feasibility of microsatellites for auroral research. The overall technical responsibility of the Astrid-2 mission was with the Swedish Space Corporation while the overall scientific responsibility was with the Alfvén Laboratory of the Royal Institute of Technology.

Astrid-2 was launched as a piggy-back on a Kosmos-3M launcher from Plesetsk, Russia $\left(62.8^{\circ} \mathrm{N}, 40.3^{\circ} \mathrm{E}\right)$ on $10 \mathrm{De}-$ cember 1998. It was placed in a circular $83^{\circ}$ inclination orbit at $1000 \mathrm{~km}$ altitude. Relative to the Sun, the nodal regression rate was $-1.7^{\circ} /$ day, implying that all local times were covered over a 3.5 month period. Figure 1 illustrates the drift of the orbital plane with respect to local time during the mission. Astrid-2 operated for about seven months until 24 July 1999, and provided about 26 Gigabytes of data from over 3000 polar orbits.

\section{The Astrid-2 spacecraft}

The spacecraft platform, built by the Swedish Space Corporation, had the dimensions $45 \times 45 \times 30 \mathrm{~cm}$. The overall mass was $29 \mathrm{~kg}$, of which about $10 \mathrm{~kg}$ was allocated for the scientific payload. The spacecraft was spin-stabilized with a nominal spin rate of $10 \mathrm{rpm}$. There were six solar panels, two 


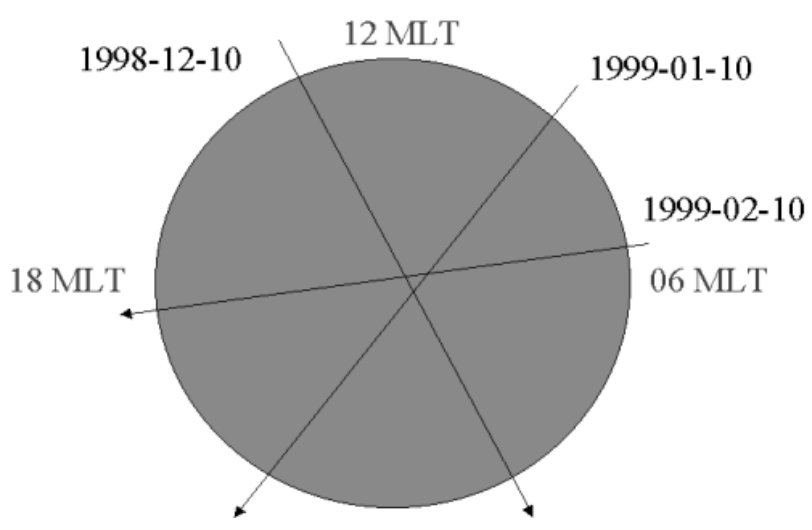

Fig. 1. Variations of the orbital plane during the mission lifetime.

fixed and four deployable, together delivering a total of $90 \mathrm{~W}$ of power when sunlit. Since the solar panels all face in the direction of the spin axis, the spacecraft was kept Sun-pointing to within $30^{\circ}$ by means of magnetorquers. The spacecraft surface, including the solar panels, was electrically conducting to avoid differential charging and to supply a sufficient area for the return current from spacecraft to plasma (caused by current biasing of the electric field sensors, and by sweeps of the electric field and plasma density instruments).

\section{The Astrid-2 scientific payload}

The scientific instruments on-board Astrid-2 together with the responsible institutions are:

- EMMA Electric and Magnetic Monitoring of the Aurora, Alfvén Laboratory, Royal Institute of Technology, Stockholm and Technical University of Denmark, Lyngby

- LINDA Langmuir INterferometry and Density experiment for Astrid-2, Swedish Institute of Space Physics, Uppsala

- MEDUSA Miniaturized Electrostatic DUal-tophat Spherical Analyzer, Swedish Institute of Space Physics, Kiruna and Southwest Research Institute, San Antonio, Texas

- PIA Photometer for Imaging the Aurora, Swedish Institute of Space Physics, Uppsala

- ASC Advanced Stellar Compass, Technical University of Denmark, Lyngby

All instruments were equipped with on-board memory, allowing the sampled data to be stored temporarily for later transmission to ground. The location of the instruments onboard the Astrid-2 platform is shown in Fig. 2. EMMA, LINDA, and MEDUSA provided excellent data throughout the seven month life time of the mission. The PIA instrument provided only one high-quality auroral image. Due to a

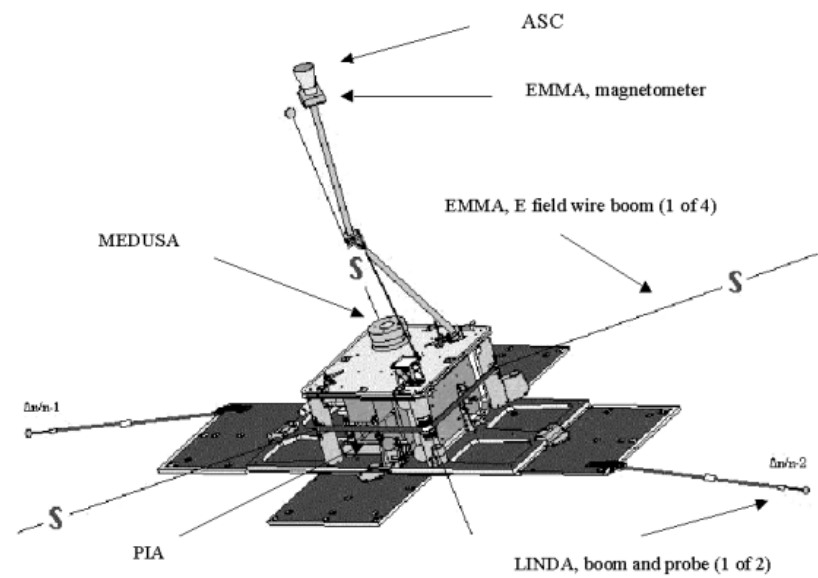

Fig. 2. The Astrid-2 scientific payload.

sensitivity decrease in the early part of the mission, no more images were provided. The ASC was flown for the first time on a rapidly spinning satellite $(7.5 \mathrm{rpm})$ which made the design very challenging. However, the ASC failed to provide attitude data during the mission (the Ørsted satellite carries a similar ASC instrument to that used on Astrid-2 but which functions well and provides excellent attitude data). Despite the lack of ASC data on Astrid-2, we could, however, retrieve the attitude (to an accuracy of half a degree) from the high-quality magnetic field measurements. Descriptions of the various instruments and their capabilities are presented in the accompanying studies of this special issue (see Holback et al., 2001; Norberg et al., 2001).

\section{Mission objectives - scientific and technological}

Astrid-2 had two primary mission objectives, both of which were met successfully. On the one hand it was a sophisticated research satellite, providing important new information on processes related to the aurora, as will be demonstrated by the various results presented in this special issue. On the other hand it was a technological pioneering mission, where innovative technologies and low-mass solutions for the instruments, sensors and deployment systems were used and flight tested for the first time. A new wire boom deployment mechanism, with a total weight of less than two kilograms, including four probes and wire booms, was developed to enable electric field measurements on Astrid-2 (Hellman, 1996). It is further discussed in the EMMA electric and magnetic field instrument description. Since most of the scientific instruments including the electric field instrument with the new wire boom deployment system proved to function excellently, the Astrid-2 performance served as a validation of the microsatellite concept for auroral research and for multispacecraft missions in the future. 


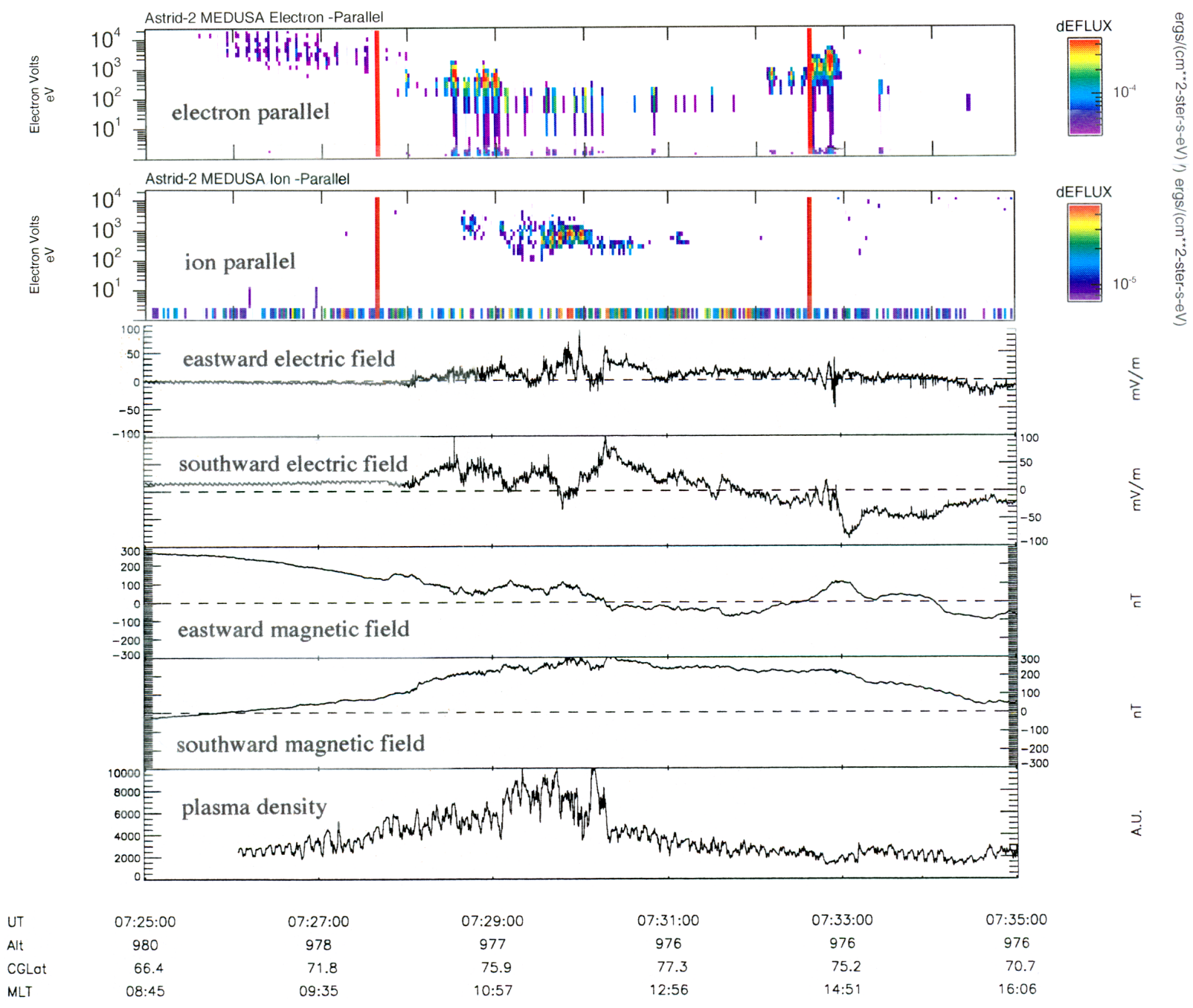

Fig. 3. Observations from a dayside orbit through the polar cusp on 7 May 1999. The panels show from top to bottom; energy time spectrograms of electrons and ions parallel to the geomagnetic field; the geomagnetic eastward and equatorward components of the electric and magnetic field, respectively; and the plasma density measured by the Langmuir probe.

\section{Ground stations and telemetry}

Two ground stations were employed for the Astrid-2 project. The primary ground station was located on the roof of the Swedish Space Corporation building in Solna, Sweden (59.3 ${ }^{\circ}$ $\left.\mathrm{N}, 18.0^{\circ} \mathrm{E}\right)$. On average the spacecraft was above the Solna horizon about $2 \mathrm{~h}$ per day during which all commands to the spacecraft were made. The second ground station was located on the South African base SANAE IV on the Antarctic continent $\left(71.1^{\circ} \mathrm{S}, 2.8^{\circ} \mathrm{W}\right)$. Because of the higher latitude, the contact time was longer at SANAE IV than at Solna. The SANAE IV station was downlink only, and thus no commanding was possible from there. The two ground stations were designed for the Astrid-2 mission to be essentially autonomous, thus not requiring manual intervention for extended periods of time. The controlling computer was remotely accessible and incoming data were time stamped using GPS time.
The telemetry used S-band, BPSK, and Viterbi-encoding, and had a net data rate of $128 \mathrm{kbits} / \mathrm{s}$ for the downlink, and $4.8 \mathrm{kbits} / \mathrm{s}$ for the uplink. With $4.5 \mathrm{~h}$ of contact time it was possible to transfer a data volume of approximately $250 \mathrm{MB}$ each day from spacecraft to ground. From the Solna station, data were accessible to the instrument groups in real-time through the Internet, and real-time commanding of the instruments was also used. Data received at SANAE were stored on DAT tapes for later transport out of SANAE by ship during the summer months. The ground station at SANAE IV was set up in collaboration with the Physics Department of the University of Natal, Durban, and was operated by their resident expedition members.

\section{First Astrid-2 results}

Astrid-2 has provided a wealth of high-quality observations of the aurora and of related phenomena. Figure 3 shows an 
example of the Astrid-2 data from a dayside orbit on 7 May 1999 crossing the pre-noon auroral oval, the polar cusp and the afternoon auroral oval. The panels show from top to bottom; energy time spectrograms of electrons and ions parallel to the geomagnetic field; the geomagnetic eastward and equatorward components of the electric and magnetic field, respectively; and the plasma density measured by the Langmuir probe. The cusp region is recognized by electron fluxes in the hundreds of eV range, ion dispersion, large-scale and superposed small-scale variations in the electric field and the magnetic field and a high plasma density. The data shown in the top and bottom panels are seen to contain a residual spin modulation.

The results of the scientific analysis of the Astrid-2 data obtained so far are described in the accompaning papers and very briefly mentioned here. The Astrid- 2 results confirm earlier Freja discoveries that significant auroral particle acceleration occurs occasionally at or below $1000 \mathrm{~km}$, in particular within the auroral return current region, although clearly much less frequent than at higher altitudes. This is supported by observations of upward $\mathrm{keV}$ electron beams, very intense electric fields $(>1 \mathrm{~V} / \mathrm{m})$ and ion heating. Possible source regions of ion clouds observed by the Astrid- 2 particle detector are discussed by Høymork et al. (2001). Full-orbit data of the plasma density in the top-side ionosphere indicate the presence of large-, medium-, and small-scale plasma density cavities on auroral field lines, of which the latter are likely to be a manifestation of the low-altitude part of the acceleration region. Statistical analysis of electric and magnetic field data shows the frequent occurrence of Alfvénic activity on auroral field lines sometimes accompanied by dispersed field-aligned electrons accelerated locally by the wave electric field (Ivchenko et al., 2001). Astrid-2 and ground-based observations from a polar crossing of the nighttime auroral bulge reveal a scenario different to that of the Cowling current model for the current closure of the bulge (Marklund et al., 2001). Astrid-2 data have also been used to study the magnetospheric response to the solar wind, indicating both a direct and a delayed response (Eriksson et al., 2001). Magnetic field data from Astrid-2 are of excellent quality and have been used for detailed modelling of the Earths magnetic field and to reveal ULF activity on auroral field lines.

Acknowledgements. The Astrid-2 project was funded by the Swedish National Space Board and by corresponding agencies in the other participating countries. The Antarctic ground station was funded through donations from the Wallenberg Foundation and from the Royal Institute of Technology. We wish to acknowledge the many scientists and engineers who have contributed to this project in different ways.

Topical Editor G. Chanteur thanks F. Mozer and G. Haerendel for their help in evaluating this paper.

\section{References}

Barabash, S., C:son Brandt, P., Norberg, O., Lundin, R., Roelof, E. C., Chase, C., and Mauk, B., Energetic neutral atom imaging by the Astrid microsatellite, Adv. Space Res., 20, (4/5), 1055-1060, 1997.

Barabash, S., Norberg, O., Lundin, R., Olsen, S., Lundin, K., C:son Brandt, P., Roelof, E. C., Chase, C., Mauk, B., Koskinen, H., and Rynö, J., Energetic neutral atom imager on the Swedish microsatellite Astrid, AGU monograph on Measurement Techniques in Space Plasmas - Fields, Geophysical Monograph 103, Eds. R. F. Pfaff, J. E. Borovsky, and D. T. Young, American Geophysical Union, 257-262, 1998.

Blomberg, L. G., Marklund, G. T., Lindqvist, P.-A., and Bylander, L., Astrid-2: An advanced auroral microprobe, in Microsatellites as Research Tools, COSPAR Colloquia Series, Vol. 10, Elsevier, 10, 57-65, 1999.

C:son Brandt, P., Barabash, S., Norberg, O., Lundin, R., Roelof, E. C., and Chase, C. J., ENA imaging from the Swedish microsatellite Astrid, J. Geophys. Res., 104, (A2), 2367-2379, 1999.

Eriksson, S., Blomberg, L. G., Ivchenko, N., Karlsson, T., and Marklund, G. T., Magnetospheric response to the solar wind as indicated by the cross-polar potential drop and the low-latitude asymmetric disturbance field, Ann. Geophysicae, 19, 649-653, 2001 (this issue).

Hellman, H., Design of wire boom system for a satellite, Royal Institute of Technology, Division of Plasma Physics, Internal Report, ALP-1996-101, 1996.

Holback, B., Jacksén, Å., Åhlén, L., Jansson, S.-E., Eriksson, A. I., Wahlund, J.-E., Carozzi, T., and Bergman, J., LINDA the Astrid-2 Langmuir probe instrument, Ann. Geophysicae, 19, 601-610, 2001 (this issue).

Høymork, S. H., Yamauchi, M., Ebihara, Y., Narita, Y., Norberg, O., and Winningham, D., Dense ion clouds of 0.1-2 keV ions inside the CPS-region observed by Astrid-2, Ann. Geophysicae, 19, 621-631, 2001 (this issue).

Hultqvist, B., The Swedish satellite project Viking, J. Geophys. Res., 95, 5749, 1990.

Ivchenko, N., Facciolo, L., Lindqvist, P. A., Kekkonen, P., and Holback, B., Disturbance of plasma environment in the vicinity of the Astrid-2 microsatellite, Ann. Geophysicae, 19, 655-666, 2001 (this issue).

Keith, W. R., Winningham, J. D., and Norberg, O., A new, unique signature of the true cusp, Ann. Geophysicae, 19, 611-619, 2001 (this issue).

Lundin, R., Haerendel, G. and Grahn, S., The Freja science mission, Space Sci. Rev., 70, 405, 1994a.

Lundin, R., Haerendel, G. and Grahn, S., The Freja project, Geophys. Res. Lett., 21, 1823, 1994b.

Marklund, G., Blomberg, L. G., Bylander, L., and Lindqvist, P.A., Astrid-2: A low-budget microsatellite mission for auroral research, ESA SP-397, 387, 1997.

Marklund, G. T., Karlsson, T., Eglitis, P., and Opgenoorth, H., Astrid-2 and ground-based observations of the auroral bulge in the middle of the nightside convection throat, Ann. Geophysicae, 19, 633-641, 2001 (this issue).

Norberg, O., Barabash, S., Sandahl, I., Lundin, R., Lauche, H., Koskinen, H., C:son Brandt, P., Roelof, E., Andersson, L., Eklund, U., Borg, H., Gimholt, J., Lundin, K., Rynö, J., and Olsen, S., The microsatellite Astrid, Proc. of the 12th ESA Symp. on European Rocket and Balloon Programmes and Related Research, Lillehammer, Norway, 29 May - 1 June, 1995.

Norberg, O., Winningham, J. D., Lauche, H., Keith, W., Puccio, W., Olsen, J., Lundin, K., and Scherrer, J., The MEDUSA electron and ion spectrometer and the PIA ultraviolet photometers on Astrid-2, Ann. Geophysicae, 19, 593-600, 2001 (this issue). 